\title{
The relationship between demographics and the academic achievement of engineering students
}

\section{Luwes, Nicolaas Johannes and Swart, James}

Department of Electrical, Electronic and Computer Engineering, Central University of Technology, Free State (CUT), South Africa.

\begin{abstract}
The changing structure of student populations or cohorts over decades' produces changing academic achievements or results. This may be due to a number of factors, including the school education system, the political system and the sociocultural system. The aim of this paper is to analyse the relationship between student demographics and the academic achievement of undergraduate engineering students over a 15-year period. A descriptive study is used to determine the relationships between specific variables that existed between 1998 and 2013. These variables include gender, age and home languages of students that are contrasted to their final grade in a compulsory Design Projects module. Students need to obtain more than 50\% to successfully complete this module, with the results indicating greater success for students with an Afrikaans or IsiZulu mother tongue than compared to students with a Sesotho, Setswana or Xhosa mother tongue. Younger students, less than 21 years of age, have a higher pass rate than older students who are more than 24 years of age. Finally, males outnumber females by more than 3:1. However, their final overall pass rates differ by only 3\%, suggesting that both genders performed equally well in the Design Projects module. A key recommendation is to provide additional academic support to older students who may be struggling to synthesize knowledge and skills from a wide number of modules.
\end{abstract}

Keywords: culture, mother tongue, ages, gender, cognitive behavioural 


\section{Introduction}

Confucius, a Chinese teacher, editor, politician, and philosopher once stated "By nature men are nearly alike; by practice men are wide apart" (Quotes, 2017). He was of the view that all infants are similar at birth and that one's surroundings influence one to become an individual in a specific cultural group (Leveridge, 2008). More recent literature continues this discourse, noting that individuals are constantly shaped by their environment (VanderStel, 2014).

The careful compilation of student profiles may help to better understand students and their personal circumstances (Nel \& Wilkinson, 2001), while serving as an early warning system (Stephens \& Myers, 2013). Student profiles have also helped to tailor teaching practices to student identity and student strengths (Hoskyn, 2013). Student demography may further interact with teacher demography and may be jointly dependent with teacher experience (Tobe, 2009). Understanding the relationship between the demography of students and their academic achievement may therefore assist academics to improve their teaching practice.

The aim of this paper is to analyse the relationship between student demographics and the academic achievement of undergraduate engineering students in a design based module, Design Projects 3 (DP3). This is done for a 15-year period from 1998 to 2013, in order to try and understand how the student population and student academic achievement has changed in South Africa (SA) that faced political and economic changes during this time.

The paper firstly discusses student demographics (gender, age and language) in relation to academic achievement. The context and assessment of DP3 is then outlined followed by the research methodology. Results are presented in two tables and then conclusions are drawn.

\section{Student demographics' impact on academic achievement in higher education}

Gender, age and language may impact on student academic achievement in higher education. A possible reason for this is that these factors influence human cognitive behaviour. The cognitive behavioural theory states that the three cornerstones namely thought, emotion and behaviour is co-dependent. For example, if one does a lot of engineering work (behaviour), one would think more about engineering (thought) and that would make one feel more like an engineer (emotion) and vice versa. This cognitive behaviour may have a negative result if stereotyping is introduced as stereotyping and cognitive behaviour is linked (Hamilton, 2015). For instance, if stereotyping influences a woman to think that she will not make a good engineer then thought and actual behaviour will be influenced. 
Contradicting results in the relationship between gender and academic achievement might be a factor of the level of gender inequality in that particular society. Factors that seem to influence gender deviations includes stereotyping (Barbuto Jr, Fritz, Matkin, \& Marx, 2007). Contradicting conclusions are seen in research that some show men perform better than women in certain fields and other times women outperform men. Others found no evidence of academic performance by gender (Richardson \& Woodley, 2003; Mlambo, 2012).

Stereotyping is also indicated as a cognitive behaviour theory factor in age (Beck, 2006). In South Africa students should typically start at 19 years of age after completed their secondary education with a National Senior Certificate at an average age 18 years (Kruger \& Sonono, 2016). Institutions of higher education have been viewed as facilities for qualified young people leaving secondary schools. However, with worldwide changes in educational policies, there seems to be a global increase in mature admissions. (Richardson \& Woodley, 2003; Mlambo, 2012). Richardson and Woodley (2003) noticed from the literature that, in the UK, older students tended to do slightly poorer than younger students in the older universities and slightly better in polytechnics and colleges of higher education. Richardson (1994) further observed that mature students were more likely adopt a deep approach and a meaning orientation, while younger students adopted a surface approach or a reproducing orientation.

Cultural and environmental elements might have an influence on cognitive behaviour. A study showed that one's environment and culture impacts on the way an individual will learn, which correlates with old philosophies that infants are similar and that their surroundings influence their development (VanderStel, 2014). In SA, a language is often an indicator of a specific culture. A key challenge, in this regard, is the a multi-cultural and multilingual society in SA where 11 official languages exist. Education is primarily done in English, which results in the majority of students studying in their second or third language that puts students at a disadvantage (Jaffer, Ng'ambi, \& Czerniewicz, 2007). The University of Cape Town revealed a $20 \%$ difference between the success rate of English first language and English second language students in 2002 (Spiegel et al., 2003). Swart and Kuriakose (2014) revealed that students from underdeveloped urban areas speaking Sesotho, Tsonga, Tswana and Zulu were among the majority of students who did not complete a module relating to work integrated learning. The effect of studying in one's mother tongue may seem to have a greater impact on one's thoughts, emotions and behaviour. 


\section{Context of this study}

DP3 is a compulsory offering for the National Diploma: Engineering: Electrical qualification that requires a National Senior Certificate (including a minimum number of credits). This module is offered at the end of the course and requires students to synthesize their previously acquired knowledge in the design and construction of a working electronic project to address a specific engineering problem. Most engineering problems require a significant amount of background knowledge from a variety of fields (DiBenedetto, Hoerl, $\&$ Snee, 2014). Students have to therefore demonstrate a number of different graduate attributes, such as engineering knowledge, problem analysis, design and development of solutions, investigation, modern tool usage, teamwork, communication, project and management and finance skills (International Engineering Alliance, 2013). The purpose of DP3 is to develop a working electronic project that will help solve a specific problem in the community or in industry. Specific learning outcomes include a portfolio that consist of a proposal, schematics, printed circuit board (PCB), fault finding and presentations. Methods of assessment include formative assessment (feedback on the proposal, schematic and PCB) and summative assessment (final judgement on the presentation, portfolio and electronic project).

\section{Research Methodology}

A descriptive study is used in this research covering the time period from 1998 through 2013. This type of study has been used in the past to determine the relationships between specific variables, such as between cognitive impairment and self-management (Vehovec, Dolansky, Josephson, \& Hughes, 2015). In this study, it is used to determine the relationship between the academic achievement (considered when students obtain $50 \%$ or more) of engineering students and their respective ages, gender and home languages. No sampling technique is used as all data analysed. The total sample size is 3700 . All data was obtained from the universities' Integrated Tertiary System (ITS) department. Data relating to gender is presented separately, where home languages, age brackets, grade distributions, student number growths and final pass rates are contrasted. Home languages are listed alphabetically according to the number of languages identified. Different mother tongues may be used to indicate different and even opposite cultural backgrounds, political stands, values, and moral standards (Niu, 2016). The age brackets of the students are divided into six categories $(<21,=21,=22,=23,=24$ and $>24$ years of age $)$ not less than a third of the maximum student number should exist for any age bracket. Students with ages below 25 are typically found in DP3, as the National Diploma requires students to have completed their secondary education. Academic achievement is related to the final grades obtained by students (A. J. Swart, 2016) and is primarily considered in this study when students attain a 
final grade of $50 \%$ or more. The pass rate is calculated by dividing the number of students obtaining $50 \%$ or more by the total number of registered students per gender, age bracket and home language.

\section{Results and discussions}

Table 1 indicates the 14 home languages of the female students along with their respective pass rates per age bracket (percentage shown on the right hand side under 'Success'). Five languages, highlighted in light grey, form the major portion of the results, as their values constitute the greater number of students (at least 20 female students and 60 male students required). Table 2 presents the results of the number of male students per home language along with their respective pass rates.

Table 1. Home languages of female students with their respective pass rates per age bracket

\begin{tabular}{lllllll|ll|l|}
\hline Languages / Ages & $<21$ & $=21$ & $=22$ & $=23$ & $=24$ & $>24$ & Total & Passing & Success \\
\hline \hline Afrikaans & $86 \%$ & $64 \%$ & $100 \%$ & $50 \%$ & $100 \%$ & $67 \%$ & 32 & 24 & $75 \%$ \\
English & $100 \%$ & $67 \%$ & NA & $100 \%$ & NA & $40 \%$ & 10 & 6 & $60 \%$ \\
IsiZulu & $67 \%$ & $55 \%$ & $50 \%$ & $67 \%$ & $100 \%$ & $50 \%$ & 30 & 19 & $63 \%$ \\
Ndebele & $50 \%$ & NA & NA & NA & NA & NA & 2 & 1 & $50 \%$ \\
Other African & NA & $50 \%$ & $100 \%$ & NA & $100 \%$ & NA & 4 & 3 & $75 \%$ \\
Other European & NA & NA & NA & NA & $0 \%$ & $0 \%$ & 2 & 0 & $0 \%$ \\
Sepedi & $100 \%$ & $50 \%$ & $67 \%$ & $50 \%$ & $100 \%$ & $100 \%$ & 19 & 15 & $79 \%$ \\
Sesotho & $75 \%$ & $69 \%$ & $62 \%$ & $62 \%$ & $57 \%$ & $57 \%$ & 396 & 252 & $64 \%$ \\
Seswati & $100 \%$ & NA & NA & NA & $100 \%$ & NA & 2 & 2 & $100 \%$ \\
Setsonga & $100 \%$ & $100 \%$ & NA & NA & NA & $100 \%$ & 5 & 5 & $100 \%$ \\
Setswana & $60 \%$ & $63 \%$ & $65 \%$ & $50 \%$ & $59 \%$ & $64 \%$ & 234 & 142 & $61 \%$ \\
Venda & $67 \%$ & $0 \%$ & $33 \%$ & $0 \%$ & $33 \%$ & $56 \%$ & 24 & 10 & $42 \%$ \\
Unknown & NA & NA & NA & NA & NA & NA & 0 & 0 & NA \\
Xhosa & $71 \%$ & $65 \%$ & $71 \%$ & $46 \%$ & $67 \%$ & $68 \%$ & 95 & 62 & $65 \%$ \\
\hline \hline Total number & 147 & 175 & 149 & 123 & 71 & 190 & 855 & 54 & 855 \\
Total passing & 106 & 112 & 96 & 69 & 44 & 114 & & 541 \\
Pass percentage & $72 \%$ & $64 \%$ & $64 \%$ & $56 \%$ & $62 \%$ & $60 \%$ & & $63 \%$ \\
Fluctuations & NA & $8 \%$ & $0 \%$ & $8 \%$ & $-6 \%$ & $2 \%$ & & & $2 \%$ \\
& & & & & & & &
\end{tabular}


The relationship between demographics and the academic achievement of engineering students

Table 2. Home languages of male students with their respective pass rates per age bracket

\begin{tabular}{|c|c|c|c|c|c|c|c|c|c|}
\hline Languages / Ages & $<21$ & $=21$ & $=22$ & $=23$ & $=24$ & $>24$ & Total & Passing & Success \\
\hline Afrikaans & $88 \%$ & $77 \%$ & $80 \%$ & $58 \%$ & $63 \%$ & $52 \%$ & 559 & 406 & $73 \%$ \\
\hline English & $72 \%$ & $80 \%$ & $75 \%$ & $91 \%$ & $71 \%$ & $48 \%$ & 89 & 63 & $71 \%$ \\
\hline IsiZulu & $91 \%$ & $56 \%$ & $67 \%$ & $87 \%$ & $45 \%$ & $73 \%$ & 69 & 49 & $71 \%$ \\
\hline Ndebele & NA & $100 \%$ & $100 \%$ & NA & NA & $100 \%$ & 5 & 5 & $100 \%$ \\
\hline Other African & $100 \%$ & $100 \%$ & $100 \%$ & $100 \%$ & NA & $60 \%$ & 16 & 12 & $75 \%$ \\
\hline Other European & NA & $100 \%$ & $0 \%$ & $100 \%$ & NA & $100 \%$ & 4 & 3 & $75 \%$ \\
\hline Sepedi & $83 \%$ & $57 \%$ & $43 \%$ & $63 \%$ & $57 \%$ & $100 \%$ & 43 & 29 & $67 \%$ \\
\hline Sesotho & $67 \%$ & $63 \%$ & $60 \%$ & $68 \%$ & $59 \%$ & $67 \%$ & 1259 & 810 & $64 \%$ \\
\hline Seswati & $100 \%$ & $100 \%$ & $100 \%$ & NA & NA & $100 \%$ & 7 & 7 & $100 \%$ \\
\hline Setsonga & $80 \%$ & $71 \%$ & $50 \%$ & $100 \%$ & $50 \%$ & $63 \%$ & 30 & 20 & $67 \%$ \\
\hline Setswana & $73 \%$ & $67 \%$ & $60 \%$ & $53 \%$ & $67 \%$ & $57 \%$ & 498 & 307 & $62 \%$ \\
\hline Venda & $100 \%$ & $100 \%$ & $100 \%$ & $100 \%$ & $100 \%$ & NA & 13 & 13 & $100 \%$ \\
\hline Unknown & $100 \%$ & NA & $50 \%$ & $0 \%$ & $50 \%$ & $67 \%$ & 9 & 5 & $56 \%$ \\
\hline Xhosa & $56 \%$ & $82 \%$ & $53 \%$ & $79 \%$ & $56 \%$ & $54 \%$ & 244 & 155 & $64 \%$ \\
\hline Total number & 373 & $\overline{2567}$ & 504 & 431 & 324 & $\overline{646}$ & \multirow[t]{4}{*}{2845} & \multirow{4}{*}{1884} & 2845 \\
\hline Total passing & 276 & 397 & 330 & 287 & 196 & 398 & & & 1884 \\
\hline Pass percentage & $74 \%$ & $70 \%$ & $65 \%$ & $67 \%$ & $60 \%$ & $62 \%$ & & & $66 \%$ \\
\hline Fluctuations & NA & $4 \%$ & $5 \%$ & $-1 \%$ & $6 \%$ & $-1 \%$ & & & $2 \%$ \\
\hline
\end{tabular}

The number of male students outweigh those of the female students by more than 3:1 (2845 male students from Table 2 versus 855 female students from Table 1). A possible reason for this may be related to stereotyping, where the cognitive behaviour of women are influenced by what society in general promotes (Cadaret, Hartung, Subich, \& Weigold, 2016). However, a very similar ratio exists with regard to the home languages of both genders, with the notable exception of Afrikaans (559 males to 32 females representing a ratio of more than 17:1). It is noteworthy that both females and males have a very similar pass rate percentage (63\% from Table 1 compared to $66 \%$ from Table 2).

The 24 years' age bracket students are the largest cohort with 190 females from Table 1 and 646 males from Table 2. However, if the majority started their National Diploma at 19 years of age (having obtained their National Senior Certificate at 18 years of age), then they would normally register for DP3 at 21 years of age, as DP3 is normally offered in the fourth semester of study. This indicates that students are taking too long to complete their qualification within the allotted time (3 years for a National Diploma in SA). This is contributing to the poor success rate (around 20\%) currently experienced in SA, according to The Council on Higher Education (2013). This impacts negatively on the funding received by institutions from the government of SA. It is further noteworthy that the highest pass rate percentage ( $72 \%$ for females and $64 \%$ for males) occurs for students in the age bracket of 21 years or less (bottom entry). This correlates with results of Richardson and 
Woodley (2003) who stated that in the UK older students tend to perform poorer that younger students at older universities.

All of the official 11 languages used in SA were found in the data (Afrikaans, English, Sepedi, Sesotho, Seswati, Tsonga, Tswana, Venda, Xhosa and Zulu) (A.J. Swart \& Kuriakose, 2014). Three additional results, as selected by students when they registered for the module DP3, were also found, namely "Other African" (such as Shona or Chichewa), "Other European" (such as French or Portuguese) and "Unknown" (those not wanting to disclose any information). The languages with the overall best pass (success) rates are Afrikaans (75\% and $73 \%$ ) and Xhosa (65\% and 64\%), while the lowest language pass rate occurs for Setswana (61\% for females and 62\% for males) (considering only languages with female numbers above 20 and male numbers above 60). This correlates well with the results from Swart and Kuriakose (2014) who found that Xhosa speaking students had one of the lowest failure rates in a module relating to work integrated learning.

The bottom entry (Fluctuations) in Table 1 and Table 2 gives the difference between pass rates for each successive age bracket. It seems to suggest that males and females our out of sync with regard to the observed fluctuations (when male pass rates increase, female pass rates decrease), except for the first fluctuation between those younger than 21 and exactly 21 years of age (4\% lower for males and $8 \%$ lower for females).

\section{Conclusions}

The aim of this paper was to analyse the relationship between student demographics and the academic achievement of undergraduate engineering students in a design based module. This was done for a 15-year period from 1998 to 2013 where the gender, age and language was analysed with regard to the academic achievement of the undergraduate students. The results revealed that males outnumber females by more than $3: 1$. However, they both enjoy a relatively modest academic pass rate of $63 \%$ and $66 \%$ respectively, suggesting that both genders perform equally well in the design based module. The results further showed that younger students, less than 21 years of age, have a higher pass rate than older students who are more than 24 years of age. Finally, students with an Afrikaans or IsiZulu mother tongue have a higher academic pass rate than compared to students with a Sesotho, Setswana or Xhosa mother tongue. A key recommendation is to provide additional academic support to older students who may be struggling to synthesize knowledge and skills from a wide number of modules. Furthermore, additional academic support should be provided to students with a Sesotho, Setswana or Xhosa mother tongue who may be struggling to understand the English language in terms of what is really expected from them in the design based module. This challenge may be alleviated through the inclusion of numerous artworks that are familiar to these students, and which may better help to explain the 
intended learning outcomes of the module. These interventions may help to better shape future engineering students, so that their engineering practice may not be miles apart, but similar to widely accepted standards that may contribute to the socio-economic development of communities, industries and countries.

\section{References}

Barbuto Jr, J. E., Fritz, S. M., Matkin, G. S., \& Marx, D. B. (2007). Effects of gender, education, and age upon leaders' use of influence tactics and full range leadership behaviors. Sex Roles, 56(1-2), 71-83.

Beck, J. S. (2006). Cognitive-behavioral therapy. Prim. Psychiatr, 13(4), 31-34.

Cadaret, M. C., Hartung, P. J., Subich, L. M., \& Weigold, I. K. (2016). Stereotype threat as a barrier to women entering engineering careers. Journal of Vocational Behavior.

Council on Higher Education. (2013). VitalStats Public Higher Education. Pretoria

DiBenedetto, A., Hoerl, R. W., \& Snee, R. D. (2014). Solving Jigsaw Puzzles. http://asq.org/quality-progress/2014/06/statistics-roundtable/jigsaw-puzzles.html

Hamilton, D. L. (2015). Cognitive processes in stereotyping and intergroup behavior: Psychology Press.

Hoskyn, M. (2013). Foreign Languages for Everyone: How I Learned to Teach Second Languages to Students with Learning Disabilities by IB Konyndyk (review). The Canadian Modern Language Review/La revue canadienne des langues vivantes, 69(2), 232-233.

International Engineering Alliance. (2013, 21 June 2013). Graduate Attributes and Professional Competencies. Retrieved 20 August, 2015, from http://www.ieagreements.org.

Jaffer, S., Ng'ambi, D., \& Czerniewicz, L. (2007). The role of ICTs in higher education in South Africa: One strategy for addressing teaching and learning challenges. International journal of Education and Development using ICT, 3(4).

Kruger, S., \& Sonono, E. (2016). " Black gold" grade12-learners: relationship between leisure/sport and satisfaction with life. South African Journal for Research in Sport, Physical Education and Recreation, 38(2), 113-127.

Leveridge, A. N. (2008). The Relationship Between Language \& Culture and the Implications for Language Teaching. Retrieved on October, 14, 2013.

Mlambo, V. (2012). An analysis of some factors affecting student academic performance in an introductory biochemistry course at the University of the West Indies. The Caribbean Teaching Scholar, 1(2).

Nel, G., \& Wilkinson, L. (2001). Where is the Any Key, Sir? Experiences of an African Teacher-To-Be. Paper presented at the Society for Information Technology \& Teacher Education International Conference.

Niu, Z. (2016). Learning a New Language, Taking up a New Culture: Language and Culture Differences between Scottish Highland and Lowland in the 18th Century 
Reflected in RL Stevenson's Kidnapped. Studies in English Language Teaching, 4(4), 573.

Quotes. (2017). Homepage. Retrieved 9 January 2017, 2017, from http://www.quotes.net/

Richardson, J. T. (1994). Mature students in higher education: Academic performance and intellectual ability. Higher Education, 28(3), 373-386.

Richardson, J. T., \& Woodley, A. (2003). Another look at the role of age, gender and subject as predictors of academic attainment in higher education. Studies in Higher Education, 28(4), 475-493.

Spiegel, M., Khotseng, B., Gxilishe, S., Kaschula, R., Van der Merwe, C., Ramesar, R., .. . Wijsenbeek, B. (2003). Towards a Language Plan for University of Cape Town: 20052010. A document prepared by Curriculum Working Group of the University of Cape Town.

Stephens, C., \& Myers, F. (2013). Signals from the Silent: Online Predictors of Nonsuccess in Business Undergraduate Students. Business and Management Education in HE, DOI: 10.11120/bmhe.12013.00005. doi: 10.11120/bmhe.2013.00005

Swart, A. J. (2016). Distance Learning Engineering Students Languish Under ProjectBased Learning, But Thrive in Case Studies and Practical Workshops. IEEE Transactions on Education, 59(2), 98-104.

Swart, A. J., \& Kuriakose, R. B. (2014). Profiles of students registered for work integrated learning at a university of technology over a 16 year period - Are there any concerns? Paper presented at the ICEIM 2014, International Conference on Education and Information Management, Durban University of Technology, Durban.

Tobe, P. F. (2009). Value-added models of teacher effects International handbook of research on teachers and teaching (pp. 1113-1134): Springer.

VanderStel, A. (2014). The Impact of Demographics in Education.

Vehovec, A. M., Dolansky, M. A., Josephson, R., \& Hughes, J. W. (2015). Compensatory Strategies Improve Medication Adherence in Heart Failure Patients With Mild Global Cognitive Impairment. Circulation, 132(Suppl 3), A19495-A19495. 